\title{
1 Fuzzy Quantification of Common and Rare Species in Ecological Communities
}

\section{2 (FuzzyQ)}

3 Juan A. Balbuena ${ }^{1}$, Clara Montlleó ${ }^{1}$, Cristina Llopis-Belenguer ${ }^{1}$, Isabel Blasco-Costa ${ }^{2}$,

4 Volodimir L. Sarabeev ${ }^{3}$, Serge Morand ${ }^{4,5}$

$5{ }^{1}$ Cavanilles Institute of Biodiversity and Evolutionary Biology, University of Valencia,

$6 \quad$ Valencia, Spain

$7 \quad{ }^{2}$ Department of Invertebrates, Natural History Museum of Geneva, Geneva,

8 Switzerland

$9{ }^{3}$ Department of Biology, Zaporizhzhia National University, Zhaporizhzhia, Ukraine

$10{ }^{4}$ Institut des Sciences de l'Evolution, CNRS, CC065, Université de Montpellier,

11 Montpellier Cedex, France; CIRAD ASTRE

$12 \quad{ }^{5}$ Faculty of Veterinary Technology, Kasetsart University, 10400 Bangkok, Thailand

13 Correspondence: Juan A. Balbuena Email: j.a.balbuena@uv.es

15 Running headline: Fuzzy Commonness and Rarity in Communities 


\section{Abstract}

1. Most species in ecological communities are rare whereas only a few are common.

This distributional paradox has intrigued ecologists for decades but the interpretation of species abundance distributions remains elusive.

2. We present Fuzzy Quantification of Common and Rare Species in Ecological

Communities (FuzzyQ) as an R package. FuzzyQ shifts the focus from the prevailing species-categorization approach to develop a quantitative framework that seeks to place each species along a rare-commonness gradient. Given a community surveyed over a number of sites, quadrats, or any other convenient sampling unit, FuzzyQ uses a fuzzy clustering algorithm that estimates a probability for each species to be common or rare based on abundance-occupancy information. Such as probability can be interpreted as a commonness index ranging from 0 to 1 . FuzzyQ also provides community-level metrics about the coherence of the allocation of species into the common and rare clusters that are informative of the nature of the community under study.

3. The functionality of FuzzyQ is shown with two real datasets. We demonstrate how FuzzyQ can effectively be used to monitor and model spatio-temporal changes in species commonness, and assess the impact of species introductions on ecological communities. We also show that the approach works satisfactorily with a wide range of communities varying in species richness, dispersion and abundance currencies.

4. FuzzyQ produces ecological indicators easy to measure and interpret that can give both clear, actionable insights into the nature of ecological communities and provides a powerful way to monitor environmental change on ecosystems. Comparison among communities is greatly facilitated by the fact that the method is relatively independent of the number of sites or sampling units considered. Thus, we consider FuzzyQ as a potentially valuable analytical tool in community ecology and conservation biology. 
41 Key-Words: Rarity, commonness, abundance-occupancy distributions, fuzzy clustering, community ecology, assembly rules, environmental monitoring.

Introduction

Ecological communities are formed by species that differ widely in abundance. Almost invariably the observation is that most species are rare, whereas a few are common (Magurran \& Henderson, 2011). This pervasive pattern has intrigued ecologists for decades but, despite the large literature on the topic, the interpretation of species abundance distributions remains elusive (Werner et al., 2014; Enquist et al., 2019). The assumption often made is that underlying factors, such as immigration, succession and competition, eventually determine differences in establishment and persistence of each species in the community (McGill et al., 2007; McGill, 2011; Alroy, 2015; Calatayud et al., 2019).

A quantitative framework for species commonness and rarity amenable to hypothesis testing and statistical modelling would facilitate evaluating the roles played by demographic variables and species traits, thereby illuminating assembly rules in ecological communities. Such a framework would also be extremely valuable for conservation biology in at least three important aspects: (1) Species rarity is an important predictor of extinction risk because the impact of environmental disturbances is expected to be higher on small populations (Davies, Margules, \& Lawrence, 2000) and since budgets for biodiversity conservation are limited, quantifying the rarity of species would facilitate prioritizing some over the others. (2) From a functional perspective, the role played by common and rare species in providing ecosystem services is currently under scrutiny. Although intuitively it might be assumed that most of the ecosystem functionality should reside in the former, the contribution of rare species is still poorly understood and, in fact, might be substantial (Leitão et al., 2016; 
Violle et al., 2017; Dee et al., 2019). So assessing rarity could also be justified in terms of identifying species that provide essential ecosystem services (Flather \& Sieg, 2007;

Violle et al., 2017; Dee et al., 2019) or stabilize ecological communities (Calatayud et al., 2019). (3) Monitoring variation of commonness-rarity patterns over time or along geographical and environmental gradients provides a simple way to obtain crucial information on ecosystem changes (McGill, 2011). For instance, if common species become increasingly rare in response to environmental disturbances, it might have a cascading effect on the rest of the community (Gaston \& Fuller, 2008).

Thus, metrics of commonness and rarity at species and community level would be extremely useful to unveil the architecture of ecological communities, assess the likelihood of extinction of rare species, correlate commonness or rarity with functional distinctiveness and monitor environmental change. However, a universal quantitative framework is currently lacking. A great deal of effort has been put on establishing the distribution patterns emerging from the categorization of species as common or rare (Gray, Bjørgesæter, \& Ugland, 2005; McGill et al., 2007; Antão, Connolly, Magurran, Soares, \& Dornelas, 2017). However, a major problem of fitting models to species abundance distributions has been adjusting the data to a suitable theoretical distribution (Williamson \& Gaston, 2005; McGill et al., 2007; Alroy, 2015). To some extent this is because the border between common and rare species is often blurred (Magurran \& Henderson, 2011), which has led authors to propose additional subcategories of rarity (Hanski, 1991; Yu \& Dobson, 2000; Arnan, Gaucherel, \& Andersen, 2011). Herein we propose shifting the focus from species categorization to a quantitative approach that seeks to place each species along a rare-commonness gradient. Fuzzy Quantification of Common and Rare Species in Ecological Communities (FuzzyQ) is based on the analysis of abundance-occupancy relationships 
91 (AORs), which assumes a positive relationship between local abundance and occupancy

92 (Gaston et al., 2000; Gaston \& He, 2011). Given a community surveyed over a number

93 of sites, quadrats, or any other convenient sampling unit, FuzzyQ applies a fuzzy

94 clustering algorithm (Kaufman \& Rousseeuw, 1990) that estimates a probability for

95 each species to be common or rare based on its AOR.

Although widely used, we acknowledge at the onset that abundance and/or occupancy are not the only criteria to assess species commonness and rarity (Gaston, 1994, 1997). However, the key point is that regardless of the data used, we can always use fuzzy clustering to quantify the degree of belonging of each species to the common or rare categories (or any other pre-established categorization for that matter).

We show herein that FuzzyQ produces ecological indicators easy to measure and interpret that are amenable to hypothesis testing and statistical modelling. In addition, FuzzyQ is distribution free, i.e. no a priori assumption about the distribution of species abundances is required. We illustrate the capabilities of the framework with two realworld examples involving each related and unrelated (i.e. not sharing species) communities and evaluate the effect of sample size on the estimation of commonness and rarity.

\section{Overview of FuzzyQ}

FuzzyQ is provided as an R package (R Core Team, 2020), available at https://github.com/Ligophorus/FuzzyQ, which depends on algorithms implemented in package cluster (Maechler, Rousseeuw, Struyf, Hubert, \& Hornik, 2019). We first illustrate application of FuzzyQ with a dataset of ant species (ants_Darwin_A in sampled in the Northern Territory, Australia (Arnan et al., 2011). 
117 TABLE 1 Overview of the functions implemented in package FuzzyQ

\begin{tabular}{|c|c|c|}
\hline Function & Description & Output \\
\hline \multirow[t]{13}{*}{ fuzzyq } & \multirow{13}{*}{$\begin{array}{l}\text { Determines the abundance- } \\
\text { occupancy per species of a site } \times \\
\text { species matrix. Performs fuzzy } \\
\text { clustering of common and rare } \\
\text { species based on abundance } \\
\text { occupancy. }\end{array}$} & $\begin{array}{l}\text { An object of class list and fuzzyq of three } \\
\text { objects: }\end{array}$ \\
\hline & & \$A_O \\
\hline & & $\begin{array}{l}\text { Fraction of sites occupied and the mean } \\
\text { abundance across sites per species }\end{array}$ \\
\hline & & \$Diss \\
\hline & & $\begin{array}{l}\text { Object of class dist with pairwise dissimilarities } \\
\text { among species based on their abundance and } \\
\text { occupancy }\end{array}$ \\
\hline & & $\$$ spp \\
\hline & & Three metrics for each species: \\
\hline & & $\begin{array}{l}\text { 1. Cluster membership, where } 0 \text { and } 1 \text { denote } \\
\text { allocation to the rare or common category, } \\
\text { respectively } \\
\text { 2. Silhouette width } \\
\text { 3. Commonness index }\end{array}$ \\
\hline & & \$global \\
\hline & & Community-level metrics: \\
\hline & & $\begin{array}{l}\text { 1. Average silhouette widths per cluster and } \\
\text { globally }\end{array}$ \\
\hline & & 2. Mean commonness indices per cluster \\
\hline & & 3. Normalized Dunn coefficient \\
\hline
\end{tabular}

fuzzyqBoot $\quad$ Produces $N$ replicates bootstrapping the site $\times$ species matrix by site and applies fuzzyq to each replicate.

fuzzyqCI Computes confidence intervals of the parameters computed with fuzzyqBoot. Three methods are available: percentile, bias corrected, and bias corrected and accelerated.

AOplot Plots the abundance occupancy relationship of a fuzzyq object.

sortClus Sorts species data of a matrix (columns) by the cluster allocation of a fuzzyq object. Useful to sort the output of fuzzyqBoot or fuzzyqCI by the original fuzzyq object.
A matrix of either species commonness indices (level = "spp") or community-level metrics (level = "global") of each bootstrap replicate

A matrix of lower and upper bound limits at a given confidence level (default 95\%)

A scatter plot of fraction of sites occupied by each species vs mean abundance per site. Common and rare species are distinguished by respective convex hulls

A matrix sorted by cluster matriz. Species are arranged by cluster and by increasing silhouette width within cluster

Table 1 provides an overview of the functions in package FuzzyQ. Function

119 fuzzyq takes a given site-by-species abundance matrix and performs a fuzzy clustering 
121 AORs to allocate each species into two clusters of common and rare species,

122 respectively. Since occupancy and abundance are in different scales and can come in

123 different units (for instance, the former can be reported as either number or fraction of

124 sites occupied), fuzzyq uses by default Gower's (1971) dissimilarities, which are

125 appropriate for such mixed data. Clustering is subsequently performed with function

126 fanny in cluster, which aims to minimize the objective function

$$
\sum_{v=1}^{k} \frac{\sum_{i, j=1}^{n} u_{i v}^{a} u_{j v}^{a} d(i, j)}{2 \sum_{j=1}^{n} u_{j v}^{a}}
$$

where $u_{i v}$ and $u_{j v}$ are the membership coefficients of observations (species in our case)

$i$ and $j$ to cluster $v, n$ is the number of observations, $k$ is the number of clusters (herein 2 :

common and rare), $a$ is a membership exponent (we set $a=2$ as in the original

130 formulation of Kaufman \& Rousseeuw 1990) and $d(i, j)$ is the dissimilarity between observations $i$ and $j$ (Maechler et al., 2019). Fig. 1a displays the fuzzyq allocation of species of the ant dataset to the rare and common clusters.

In fuzzy clustering, each observation can be assigned to several clusters with a different level of certainty. So $u_{i v}$ in (1) above represents the probability of the $i^{\text {th }}$ observation belonging to cluster $v$ (Kaufman \& Rousseeuw, 1990). We re-interpret these probabilities as indices of commonness $\left(C_{i}\right)$ and rarity $\left(R_{i}\right)$ for species $i$, so that each species is classified simultaneously as common and rare with a certain level of certainty. (Given that $C_{i}=1-R_{i}$, we will only report $C_{i}$ ). fuzzyqBoot generates and applies fuzzyq to bootstrap replicates by site of the species abundance matrix and fuzzyqCI computes confidence intervals of $C_{i}$ based on these replicates (Fig. 1b). 


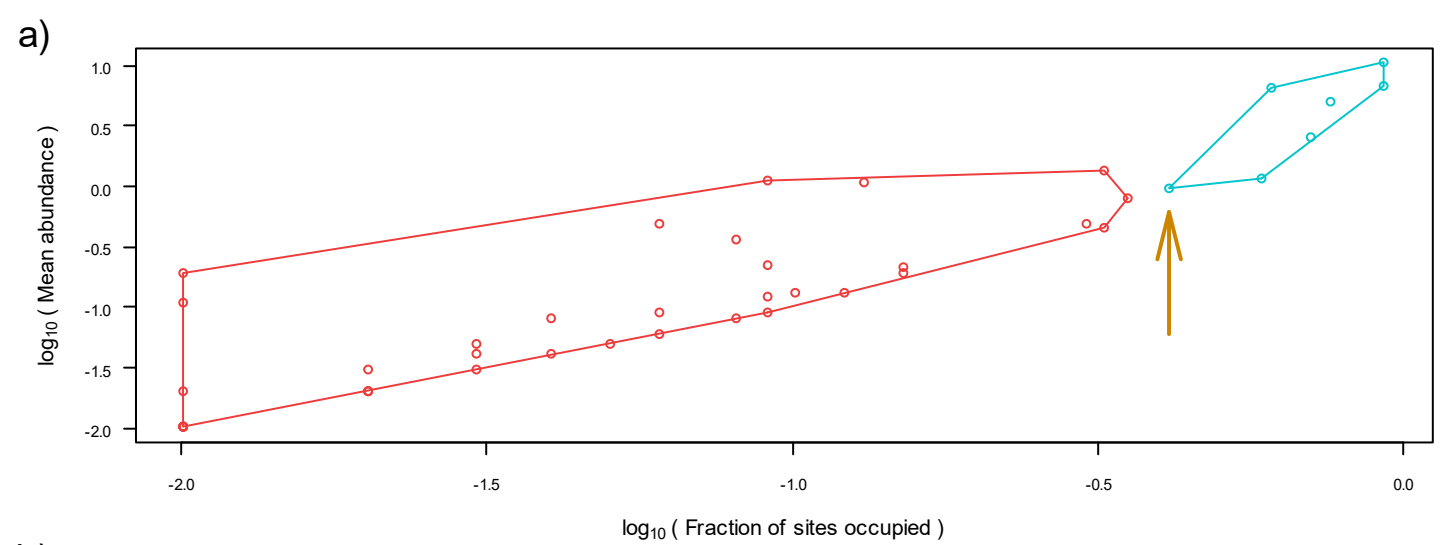

b)

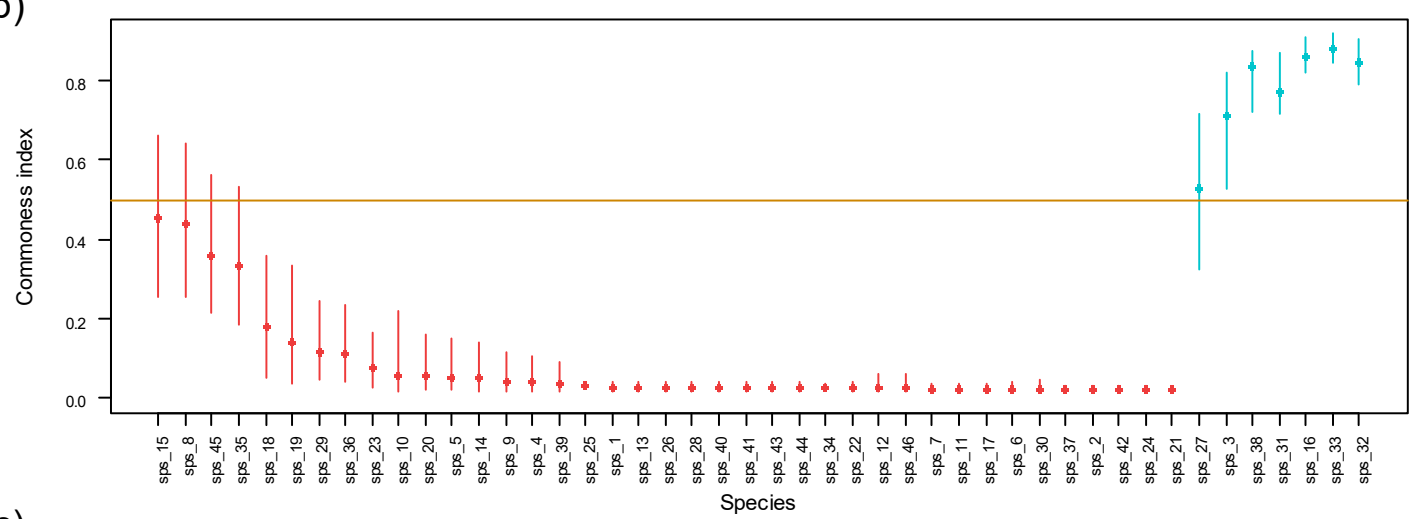

c)

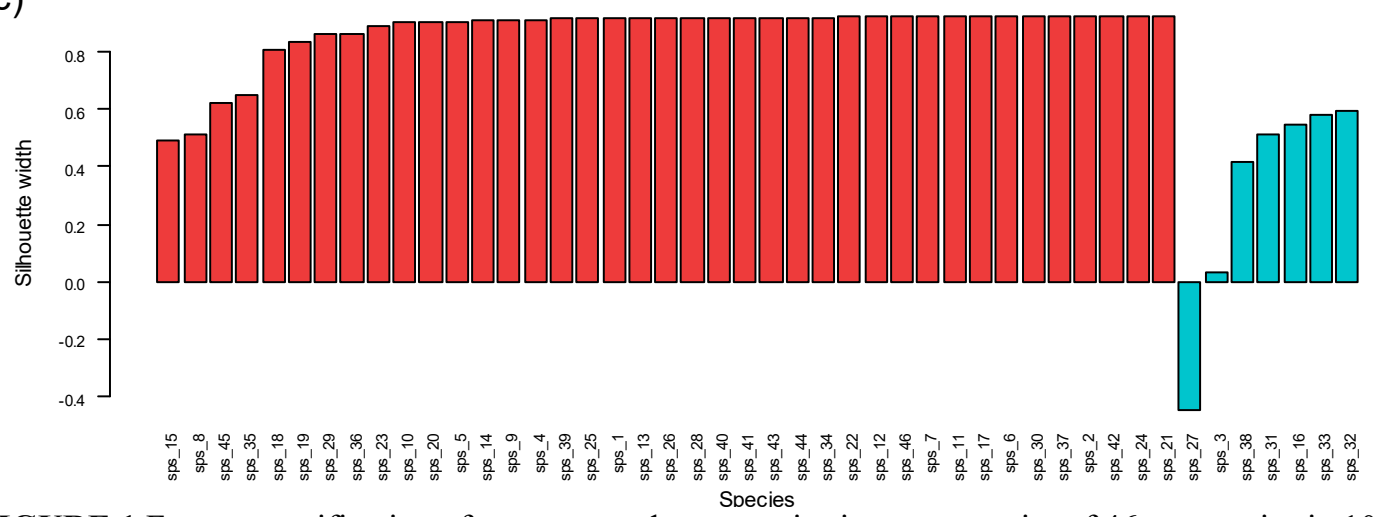

FIGURE 1 Fuzzy quantification of common and rare species in a community of 46 ant species in 100 plots (Arnan et al., 2011, Calatayud el al., 2019). (a) Abundance occupancy relationship of species.

The arrow points to the position of Species 27. (b) Commonness indices of species. Error bars represent bias-corrected and accelerated 95\% confidence intervals (error bars) (Efron \& Tibshirani, 1994) computed with 1,000 replicates bootstrapping the plots of the abundance matrix. The horizontal line marks the 0.5 threshold separating rare and common species. (c) Silhouette plot of the 46 ant species. The negative value of Species 27 suggests a poor fit to the cluster of common species. Light blue and light red denote common and rare species, respectively. 

these of species in the other cluster, as follows:

$$
s_{i}=\frac{c_{i}-r_{i}}{\max \left(c_{i}, r_{i}\right)}
$$

where $c_{i}$ and $r_{i}$ are the mean dissimilarity between species $i$ and all other species in the

clusters of common and rare species, respectively (Kaufman \& Rousseeuw, 1990). Fig.

1c shows the species silhouettes of the ant database. Silhouettes can range between -1 and +1 . The high positive values of most rare ant species indicate that they are well matched to its own cluster. Common ant species showed smaller silhouette widths, suggesting a weaker cluster. In particular, the negative silhouette of species 27 indicates a poor fit to the common-species group (Fig. 1c), which conforms to its position in the AOR plot and its $C_{i} \approx 0.5$ (Fig. 1a, b). the common- and rare-species clusters, and the strength of overall classification. The former is assessed by the average silhouettes' widths of the common and rare species ( $\bar{S}_{C}$ and $\bar{S}_{R}$, respectively) and, alternatively, by the corresponding average commonness coefficients $\left(\bar{C}_{C}\right.$ and $\left.\bar{C}_{R}\right)$. The latter can be appraised by the average silhouette width of the whole community $(\bar{S})$ and the normalized Dunn's partition coefficient $\left(D^{\prime}\right)$ (Kaufman \& Rousseeuw, 1990). The Dunn's coefficient is computed as vary between 0 (complete fuzziness) and 1 (hard clusters). When $k=2$, as in our case, the normalized Dunn's coefficient is $D^{\prime}=2 D-1$ (Kaufman \& Rousseeuw, 1990). 

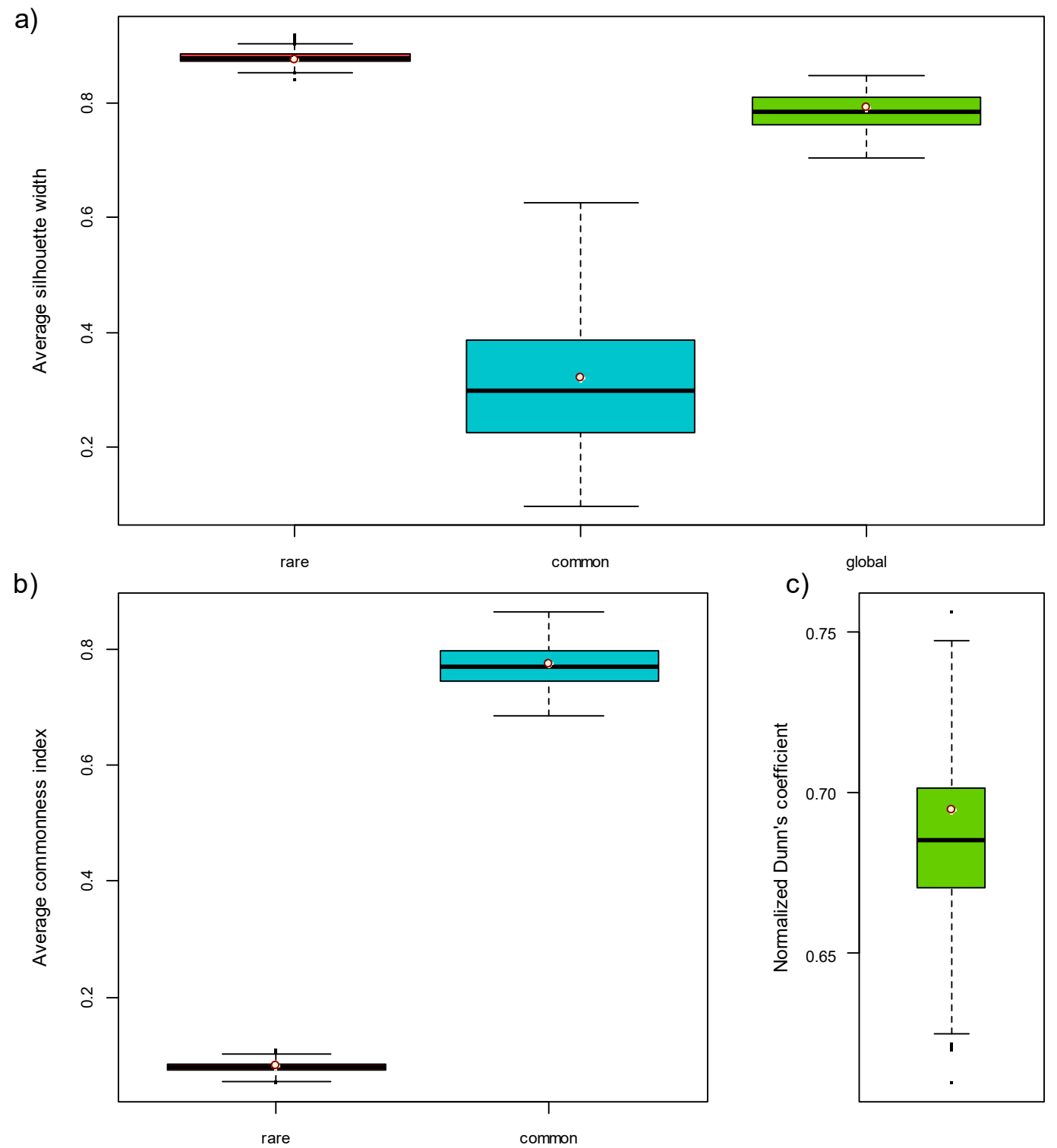

FIGURE 2 Community-level estimates (points) and their variation computed with 1,000 replicates bootstrapping the plots of the abundance matrix (boxplots) of 46 ant species in 100 plots (Arnan et al., 2011, Calatayud el al., 2019). (a) Average silhouette widths of rare, common and all species. (b) Average commonness indices of rare and common species. (c) Normalized Dunn's coefficient. Fig. 2 displays the global metrics of the ant species database. $\bar{S}$ and $D^{\prime}$ 'were 0.79 and 0.69 , which suggests a strong clustering structure separating common and rare species (Kaufman \& Rousseeuw, 1990). We assessed the variation of the global

167 estimates by bootstrapping the sites of the sites $\times$ species matrix with fuzzyqBoot.

Compared with common ones, rare species showed a higher average silhouette width, 
and showed lower variation in both silhouettes and commonness indices, indicating that they form a harder cluster (Fig. 2).

\section{Worked-out examples}

172 We demonstrate the new method and its capabilities, with two real datasets involving

173 the comparison of related and unrelated communities, respectively. When comparing

174 several communities, one must consider how to deal with species absences. Absences may be due to (a) eco-evolutionary constraints (structural absence), (b) sampling variability (random absence), or (c) methodological errors (false absences) (BlascoMoreno, Pérez-Casany, Puig, Morante, \& Castells, 2019). Although fuzzyq cannot deal with (c), it would produce different metrics in (a) and (b) situations and researchers

179 should make an informed decision based on the nature of their system. The logical argument $r m$. absent in fuzzyq specifies whether species absences are to be treated as structural or random.

\section{Example 1. Mammal Data from Powdermill Biological Station 1979-1999}

To illustrate how to monitor changes in species commonness in a community, we used a long-term (1979-1999) time series of small mammal abundances from the Powdermill Biological Station in Pennsylvania, USA (Merrit, 2013). Mammals were captured in a 1-ha live trapping grid consisting of $10 \times 10$ quadrats of trap stations at 10 -m intervals. Trapping was conducted bimonthly from September 1979 to October 1999. For the sake of demonstration, the abundance of each mammal species was aggregated per quadrat and per year in order to capture the annual variation in commonness of each species and we assumed that the pool of species did not change over the study period (random absences). 
a)

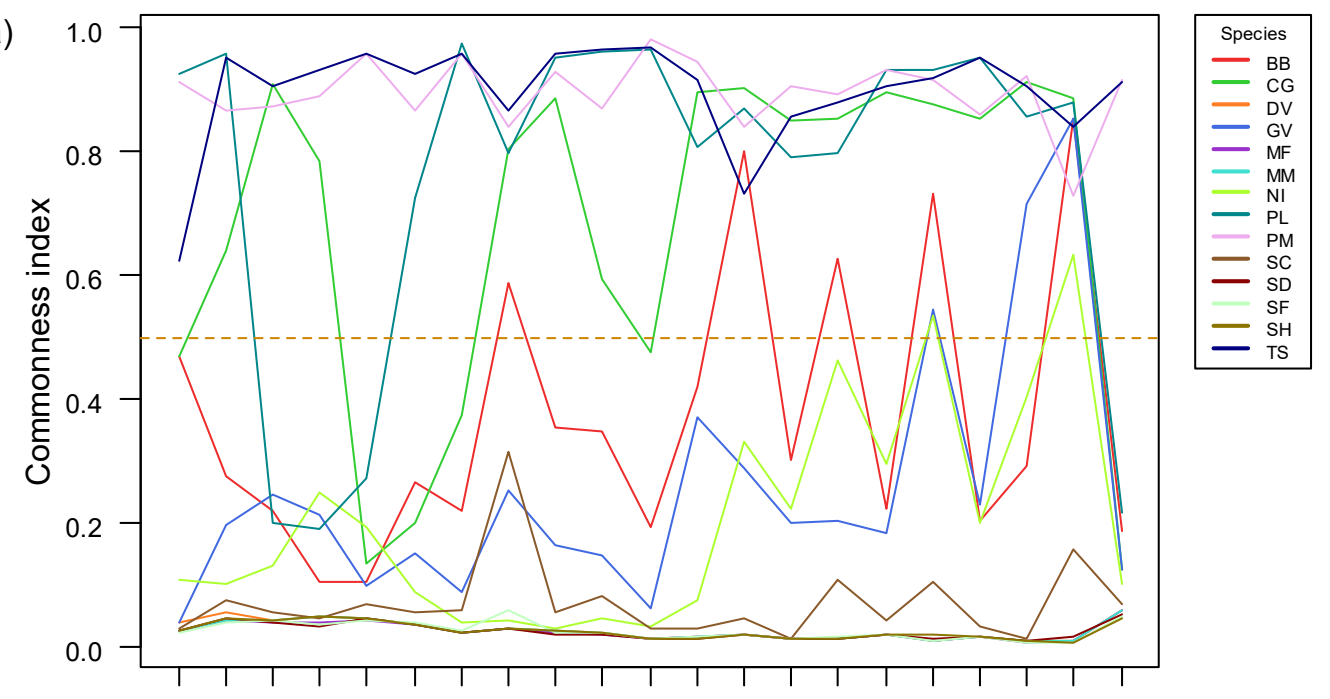

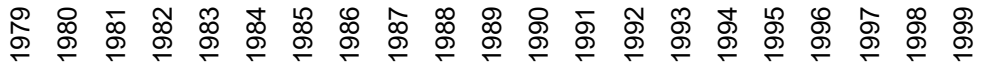

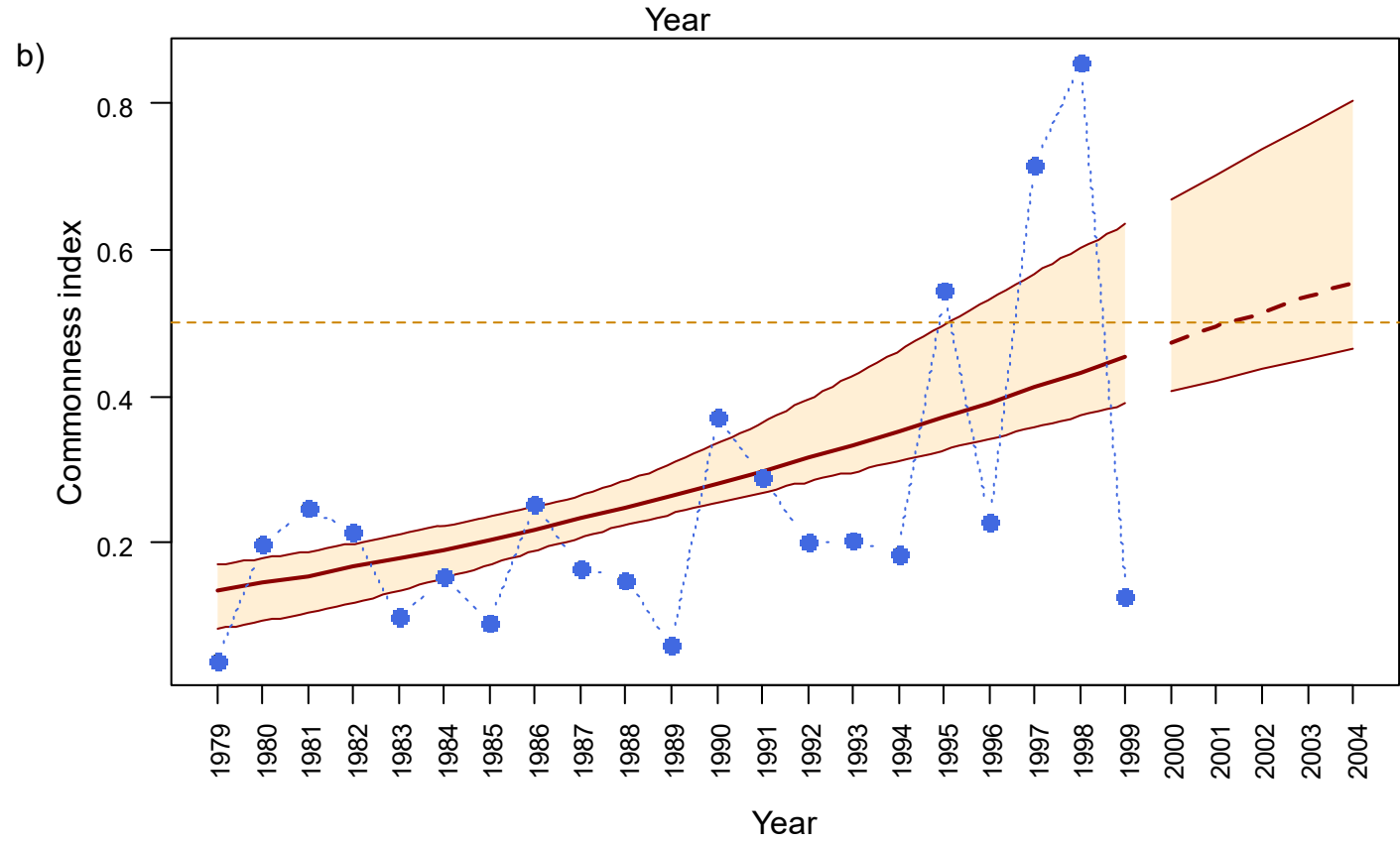

FIGURE 3 Fuzzy quantification of common and rare species in a community of 14 mammal species sampled at the Powdermill Biological Station from 1979 to 1999 (Merrit, 2013). (a) Variation of commonness indices in the study period. (b) Generalized Additive Model describing the variation in commonness of Glaucomys volans (GV) in the study period and predicted change (2001-2004). Blue points: observed values. Thick red line: fitted and predicted model (continuous and stippled lines). Thin red line: $95 \%$ confidence interval of the model. Stippled orange line: 0.5 threshold between rare and common species. Species abbreviations: BB, Blarina brevicauda; CG, Clethrionomys gapperi; DV, Didelphis virginiana; GV, Glaucomys volans; MF, Mustela frenata; MM, Marmota monax; NI, Napaeozapus insignis; PL, Peromyscus leucopus; PM, Peromyscus maniculatus; SC, Sorex cinereus; SD, Sorex dispar; SF, Sorex fumeus; SH, Sorex hoyi; TS, Tamias striatus. 

consistently as common and rare, respectively, throughout the study period. The $C_{i}$ s of

194 the remaining five varied considerably over the years (Fig. 3a). We modelled the change

195 in commonness of a species in the latter group, Glaucomys volans $(\mathrm{GV})$, by fitting a

196 Generalized Additive Model. Bootstrap replicates to fit $95 \%$ confidence intervals to the 197 model were generated with fuzzyqBoot. The fitted model was also used to predict the change in $C_{i}$ five years ahead. (Details on model fitting are given in an accompanying $\mathrm{R}$ script. See Data Availability below.) The model suggests a progressive increase in $C_{i}$ of G. volans over the years and predicts a similar increase rate in the following years (Fig. $3 b)$.

Example 2. Parasite communities of the so-iuy mullet in native and introduced areas

We compared the patterns of commonness and rarity of helminth communities of the so-iuy mullet (Planiliza haematocheilus in its native (Sea of Japan) and introduced (Sea of Azov and Black Sea) areas (Llopis-Belenguer, Blasco-Costa, Balbuena, Sarabeev, \& Stouffer, 2020). We used here 12 and 7 surveys in the introduced and native areas, respectively, in which the number of fish sampled was $\geq 20$, totalling 378 and 192 fish, respectively. Based on biogeographical evidence, species absences within the native and introduced areas were treated as random zeros (Kostadinova, 2008). 
a)

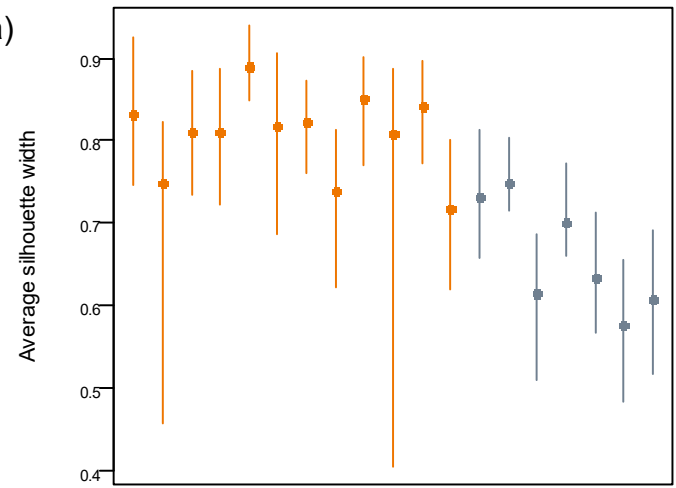

b)

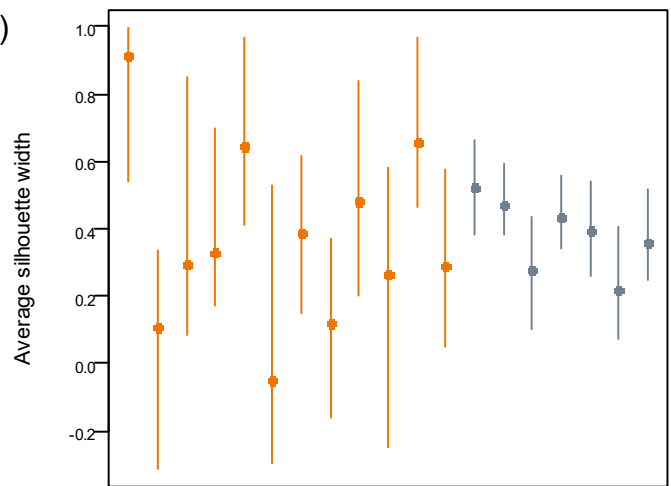

c)

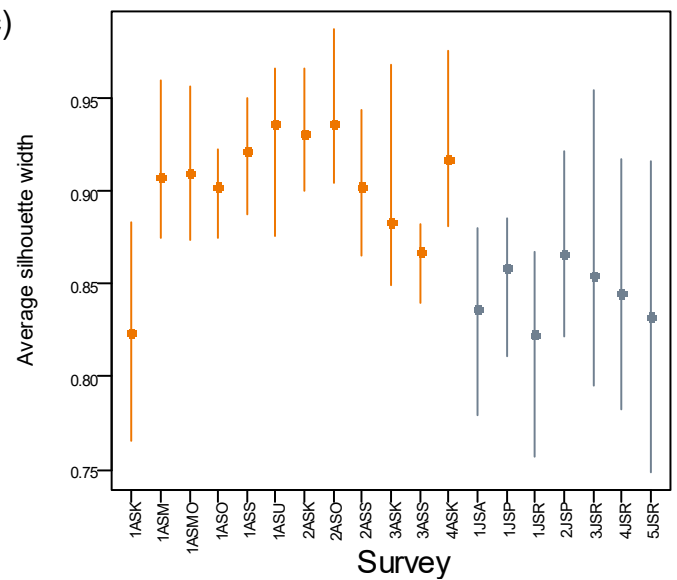

d)

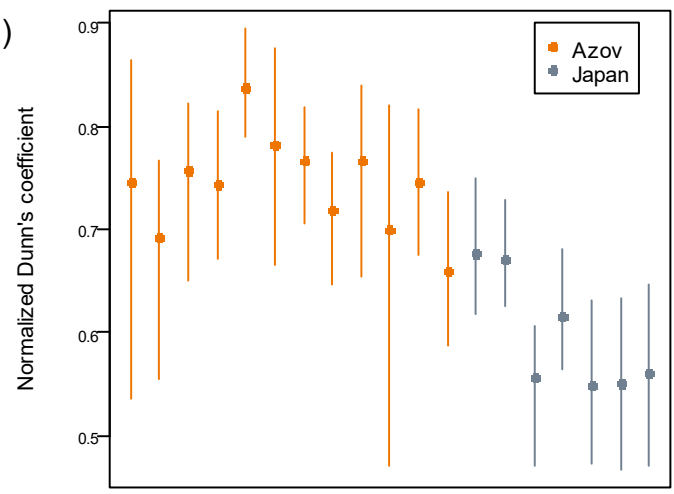

e)

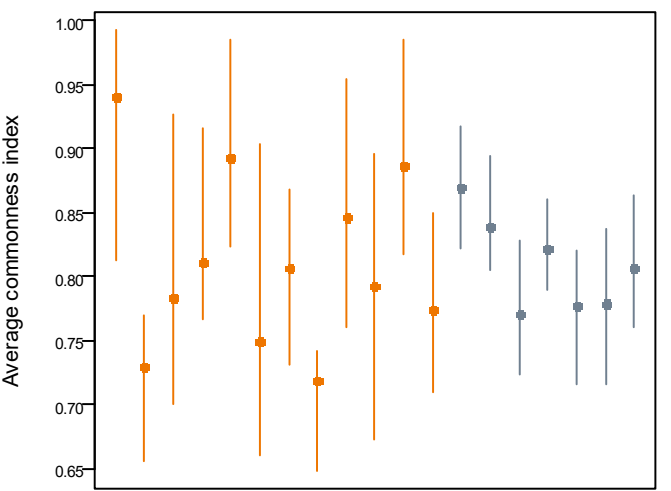

f)

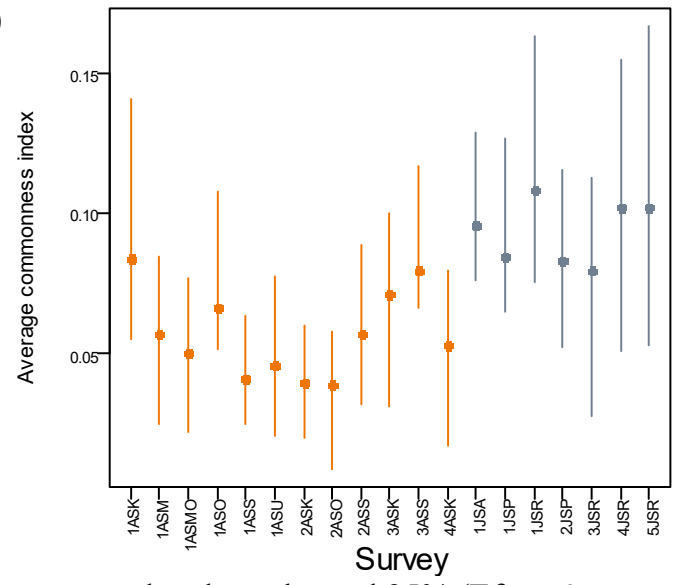

FIGURE 4 Community-level estimates (points) and bias-corrected and accelerated $95 \%$ (Efron \&

Tibshirani, 1994) confidence intervals (error bars) computed with 1,000 replicates bootstrapping the plots of the abundance matrix of helminth communities of Planiliza haematocheilus in 7 native (Japan Sea) and 12 introduced (Azov and Black Seas) surveys. (a) Average silhouette widths of all species. (b) Idem common species. (c) Idem rare species. (d) Normalized Dunn's coefficient. (e) Average commonness indices of common species. (f) Idem rare species.

211 fuzzyqBS and fuzzyqCI to estimate their 95\% confidence intervals (Fig. 4).

Differences in these metrics between surveys in the native and introduced areas were 
214 higher $\bar{S}_{R}$ and lower $\bar{C}_{R}$ than in the native one ( $p=0.0012$ and $p=0.0003$, respectively).

215 Differences in $\bar{S}$ and $D^{\prime}$ were also significant ( $p=0.0003$ and $p=0.0002$, respectively),

216 indicating a clearer distinction between common and rare species in the introduced area

217 than in the native one. By contrast, there was no evidence for significant differences

218 between areas in $\bar{S}_{C}$ and $\bar{C}_{C}(p=0.71$ and $p=0.97$, respectively). These results conform

219 to previous work that indicates that the introduction of the mullet so-iuy in the new area

220 entailed a deep structural change in its helminth communities (Sarabeev, Balbuena, \&

221 Morand, 2017; Llopis-Belenguer et al., 2020). Most native species were lost and only

222 two Ligophorus spp. common in the native area were co-introduced and remained

223 common in the introduced area (Figs. S1, S2 in Supporting Information). So the

224 majority of species in the introduced area were acquired from local grey mullet species

225 (Sarabeev et al., 2017). Since newly acquired parasite species are expected to lack

226 specific adaptations to the new host, this would account for their pronounced rarity

227 compared to rare species in the native area (Sarabeev, Balbuena, \& Morand, 2018).

228 Effect of number of sites

229 A key question for users interested in comparing different communities is whether the 230 community-level estimates depend on the number of sites sampled. We examined this

231 issue using 20 datasets compiled in Calatayud et al. (2019) and (Jeliazkov et al., 2020)

232 involving $87+$ sites. These included communities varying widely in taxonomic

233 composition, species richness and spatial scale. In addition, different abundance

234 currencies were employed. (See Table S1 in Supporting Information for details.) Being

$235 N$ the total number of sites of a given dataset, global metrics $\left(\bar{S}_{,} \bar{S}_{C}, \bar{S}_{R}, \bar{C}_{C}, \bar{C}_{R}\right.$ and $\left.D^{\prime}\right)$

236 were computed in most cases dropping successively $1,2,3, \ldots, N-10$ sites randomly

237 drawn (without replacement) from the dataset. (In species-poor communities or

238 communities with very sparsely distributed species, the series was $1,2,3, \ldots, N-20$ ). 
bioRxiv preprint doi: https://doi.org/10.1101/2020.08.12.247502; this version posted August 13,2020 . The copyright holder for this preprint (which was not certified by peer review) is the author/funder, who has granted bioRxiv a license to display the preprint in perpetuity. It is made available under aCC-BY-NC 4.0 International license.

239 Species absences in each draw were treated as structural, because our goal was to

240 examine how incomplete species coverage resulting from low sample sizes affected the

241 estimation of global metrics.


$$
-\bar{s}_{R}-\bar{s}_{C}-\bar{s}-\bar{C}_{R}-\bar{c}_{C}-D^{\prime}
$$

FIGURE 5 Variation of community-level metrics with number of sites in eight, 87+-site, databases

from Jeliazkov et al. (2020) (a-g) and Calatayud et al. (2019) (h): (a) BrindAmour2011a; (b)

Pavoine2011; (c) Jeliazkov2014; (d) Barbaro2009a; (e) Chmura2016; (f) Ribera2001; (g)

Goncalves2014a; (h) ants_data_Xavi_Darwin_A. Abbreviations: $\bar{S}_{R}$, average silhouette rare species; $\bar{S}_{C}$, idem common species; $\bar{S}$, idem all species; $\bar{C}_{R}$, Commonness coefficient rare species; $\bar{C}_{C}$, idem 
common species; D’, Normalized Dunn's coefficient. Details of these datasets are given in Table S1, Supporting Information.

Fig. 5 shows the variation of the global metrics with the number of sites in eight of the 20 datasets. (Results for the remaining 12 datasets are given in Fig. S3,

244 Supporting Information.) No clear trend of variation with the number of sites was apparent (Fig. 5 and Fig. S3, Supporting Information). Although in some datasets large

246 fluctuations occurred ( $\bar{S}_{C}$ in particular was quite labile in some examples), variation in 247 global parameters did not seem related to sample size. The results suggest that 30 to 50 sites are sufficient to yield reliable estimates although bootstrapping should be used to capture their variability.

\section{Final remarks}

251 FuzzyQ provides a new quantitative framework to study the distribution of common and rare species in ecological communities. The approach supplies simple and intuitive ecological indicators that can give both clear, actionable insights into the nature of ecological communities and a powerful way to monitor quantitatively environmental change on ecosystems. We show that the approach works satisfactorily with a wide range of communities varying in species richness, dispersion and abundance currencies. The only obvious limitation in its application is that fuzzy clustering requires that $k \geq$ $n / 2$-1 (Kaufman \& Rousseeuw, 1990). As in our case $k=2$, FuzzyQ cannot be applied to communities composed of $\leq 5$ species. In addition, the application of fuzzyqBoot in communities with low number of species can lead to a number of null replicates because of this limitation. is relatively independent of the number of sites or sampling units considered. However, the use of FuzzyQ in comparative settings comes with an important caveat. Since FuzzyQ is based on AORs and occupancy is known to vary with spatial scale (Hui, 
Veldtman, \& McGeoch, 2010; Steenweg, Hebblewhite, Whittington, Lukacs, \&

McKelvey, 2018), differences in scaling can compromise comparison among

sense. However, we cannot completely rule out that potential differences between the

native and introduced areas in fish mobility could introduce a hidden bias (Steenweg et al., 2018).

biased because species of similar occupancies at that level may have very different introducing additional/different traits when computing the dissimilarity matrix).

Therefore, we consider that FuzzyQ is a potentially valuable analytical tool in community ecology and conservation biology.

\section{Data Availability}

Table 2 provides information of the availability of the datasets used herein. $\mathrm{R}$ scripts and $\mathrm{R}$ markdown files to run the illustrative examples, and the FuzzyQ R package are available at https://ligophorus.github.io/FuzzyQ/ (**DOI no. pending**).

Acknowledgements

We thank Xavier Arman and co-workers for allowing us to incorporate their ant species dataset to the FuzzyQ package. Study funded by the Ministry of Science and Innovation, Spain 
NSF Grants BSR-8702333-06, DEB-9211772, DEB-9411974, DEB-0080381 and DEB-

0621014.

TABLE 2 Availability of the datasets used with FuzzyQ.

\begin{tabular}{lll}
\hline Datasets & Source & Reference \\
\hline $\begin{array}{l}\text { Mammals } \\
\text { Powdermill }\end{array}$ & https://doi.org/10.6073/pasta/83c888854e239a79597999895bb61cfe & Merritt (2011) \\
Helminths so-iuy & https://doi.org/10.7910/DVN/IWIKOL & Llopis-Belenguer \\
mullet & & et al. (2020) \\
$\begin{array}{l}\text { Large } \\
\text { communities }\end{array}$ & https://idata.idiv.de/ddm/Data/ShowData/286 & Jeliazkov et al. \\
$\begin{array}{l}\text { Large } \\
\text { communities }\end{array}$ & https://doi.org/10.6084/m9.figshare.9906092 & $(2020)$ \\
\end{tabular}

\section{Authors' contributions}

JAB conceived the idea. JAB and SM developed the theory and outlined the study. CM, CLB critical feedback on the drafts and gave final approval for submission.

\section{References}

Alroy, J. (2015). The shape of terrestrial abundance distributions. Science Advances, 1(8), e1500082. doi:10.1126/sciadv.1500082

Antão, L. H., Connolly, S. R., Magurran, A. E., Soares, A., \& Dornelas, M. (2017). Prevalence of multimodal species abundance distributions is linked to spatial and taxonomic breadth.

Arnan, X., Gaucherel, C., \& Andersen, A. N. (2011). Dominance and species co-occurrence in highly diverse ant communities: a test of the interstitial hypothesis and discovery of a threetiered competition cascade. Oecologia, 166(3), 783-794. doi:10.1007/s00442-011-1919-y

Blasco-Moreno, A., Pérez-Casany, M., Puig, P., Morante, M., \& Castells, E. (2019). What does

312 Calatayud, J., Andivia, E., Escudero, A., Melián, C. J., Bernardo-Madrid, R., Stoffel, M., ... 

5

Davies, K. F., Margules, C. R., \& Lawrence, J. F. (2000). Which traits of species predict population declines in experimental forest fragments? Ecology, 81(5), 1450-1461. doi:10.1890/0012-9658(2000)081[1450:WTOSPP]2.0.CO;2

Dee, L. E., Cowles, J., Isbell, F., Pau, S., Gaines, S. D., \& Reich, P. B. (2019). When do ecosystem services depend on rare species? Trends in Ecology \& Evolution, 34(8), 746-758. doi:10.1016/j.tree.2019.03.010

Efron, B., \& Tibshirani, R. J. (1994). An introduction to the bootstrap. New York, NY:

Enquist, B. J., Feng, X., Boyle, B., Maitner, B., Newman, E. A., Jørgensen, P. M., ... McGill, B. J. (2019). The commonness of rarity: Global and future distribution of rarity across land plants. Science Advances, 5(11), eaaz0414. doi:10.1126/sciadv.aaz0414

Flather, C. H., \& Sieg, C. H. (2007). Species rarity: definition, causes, and classification. In M.G. Raphael \& R. Molina (Eds.), Conservation of rare or little-known species: biological, social, and economic considerations (pp. 40-66). Washington D.C.: Island Press.

Gaston, K. J. (1994). Rarity (1st ed., Vol. 13). Dordrecht, Netherlands: Springer. doi:10.1007/978-94-011-0701-3

Gaston, K. J. (1997). What is rarity? In W.E. Kunin \& K.J. Gaston (Eds.), The biology of rarity. Causes and consequences of rare-common differences (pp. 30-47). Dordrecht, Netherlands: Springer. doi:10.1007/978-94-011-5874-9_3

Gaston, K. J., Blackburn, T. M., Greenwood, J. J. D., Gregory, R. D., Quinn, R. M., \& Lawton, J. H. (2000). Abundance-occupancy relationships. Journal of Applied Ecology, 37(s1), 3959. doi:10.1046/j.1365-2664.2000.00485.x

Gaston, K. J., \& Fuller, R. A. (2008). Commonness, population depletion and conservation biology. Trends in Ecology \& Evolution, 23(1), 14-19. doi:10.1016/j.tree.2007.11.001 
Gaston, K. J., \& He, F. (2011). Species occurrence and occupancy. In A.E. Magurran \& B.J.

Gower, J. C. (1971). A general coefficient of similarity and some of its properties. Biometrics, 27(4), 857-871. doi:10.2307/2528823

Gray, J. S., Bjørgesæter, A., \& Ugland, K. I. (2005). The impact of rare species on natural assemblages. Journal of Animal Ecology, 74(6), 1131-1139. doi:10.1111/j.13652656.2005.01011.x

Hanski, I. (1991). Single-species metapopulation dynamics: concepts, models and observations. 8312.1991.tb00549.x

He, F., \& Condit, R. (2007, July). The distribution of species: occupancy, scale, and rarity. doi:10.1017/CBO9780511814938.005

Hui, C., Veldtman, R., \& McGeoch, M. A. (2010). Measures, perceptions and scaling patterns

Jeliazkov, A., Mijatovic, D., Chantepie, S., Andrew, N., Arlettaz, R., Barbaro, L., ... Chase, J. environment and space. Scientific Data, 7, 6. doi: 10.1038/s41597-019-0344-7

Kaufman, L., \& Rousseeuw, P. J. (1990). Finding groups in data: An introduction to cluster analysis. New York, N.Y.: John Wiley \& Sons. doi:10.1002/9780470316801

Kostadinova, A. (2008). A checklist of macroparasites of Liza haematocheila (Temminck \& assemblages. Proceedings of the Royal Society B: Biological Sciences, 283(1828), 
367 Llopis-Belenguer, C., Blasco-Costa, I., Balbuena, J. A., Sarabeev, V., \& Stouffer, D. B. (2020).

368 Native and invasive hosts play different roles in host-parasite networks. Ecography, 43(4).

369 doi: $10.1111 /$ ecog. 04963

370 Maechler, M., Rousseeuw, P., Struyf, A., Hubert, M., \& Hornik, K. (2019). cluster: Cluster

371 analysis basics and extensions. R package version 2.1.0. Retrieved 29 July 2020, from https://CRAN.R-project.org/package=cluster

Magurran, A. E., \& Henderson, P. A. (2011). Commonness and rarity. In A.E. Magurran \& B.J. McGill (Eds.), Biological diversity: frontiers in measurement and assessment (pp. 97-104). Oxford, U.K.: Oxford University Press.

McGill, B. (2011). Species abundance distributions. In A.E. Magurran \& B.J. McGill (Eds.), Biological diversity: frontiers in measurement and assessment (pp. 105-122). Oxford, U.K.: Oxford University Press.

McGill, B. J., Rampal S. Etienne, John S. Gray, David Alonso, Marti J. Anderson, Habtamu Kassa Benecha, ... Ethan P. White. (2007). Species abundance distributions: moving beyond

Merritt, J. (2018). Long term mammal data from Powdermill biological station 1979-1999 [Data set]. Environmental Data Initiative.

Sarabeev, V., Balbuena, J. A., \& Morand, S. (2017). Testing the enemy release hypothesis:

R Core Team (2020). R: A language and environment for statistical computing. R Foundation for Statistical Computing, Vienna, Austria. https://www.R-project.org/. Accessed 7 August Abundance and distribution patterns of helminth communities in grey mullets (Teleostei: Mugilidae) reveal the success of invasive species. International Journal for Parasitology, 47, 687-696. doi:10.1016/j.ijpara.2017.05.006

Sarabeev, Volodimir, Balbuena, J. A., \& Morand, S. (2018). International Journal for 

scales define occupancy and underlying occupancy-abundance relationships in animals. Ecology, 99(1), 172-183. doi:10.1002/ecy.2054

398 Violle, C., Thuiller, W., Mouquet, N., Munoz, F., Kraft, N. J. B., Cadotte, M. W., ... Mouillot, D. (2017). Functional rarity: The ecology of outliers. Trends in Ecology \& Evolution, 32(5), 356-367. doi:10.1016/j.tree.2017.02.002

401 Werner, E. E., Davis, C. J., Skelly, D. K., Relyea, R. A., Benard, M. F., \& McCauley, S. J. (2014). Cross-scale interactions and the distribution-abundance relationship. PLOS ONE, 9(5), e97387. doi:10.1371/journal.pone.0097387

404 Williamson, M., \& Gaston, K. J. (2005). The lognormal distribution is not an appropriate null 405 hypothesis for the species-abundance distribution. Journal of Animal Ecology, 74(3), 409422. doi:10.1111/j.1365-2656.2005.00936.x

407 Yu, J., \& Dobson, F. S. (2000). Seven forms of rarity in mammals. Journal of Biogeography, 27(1), 131-139. doi:10.1046/j.1365-2699.2000.00366.x 